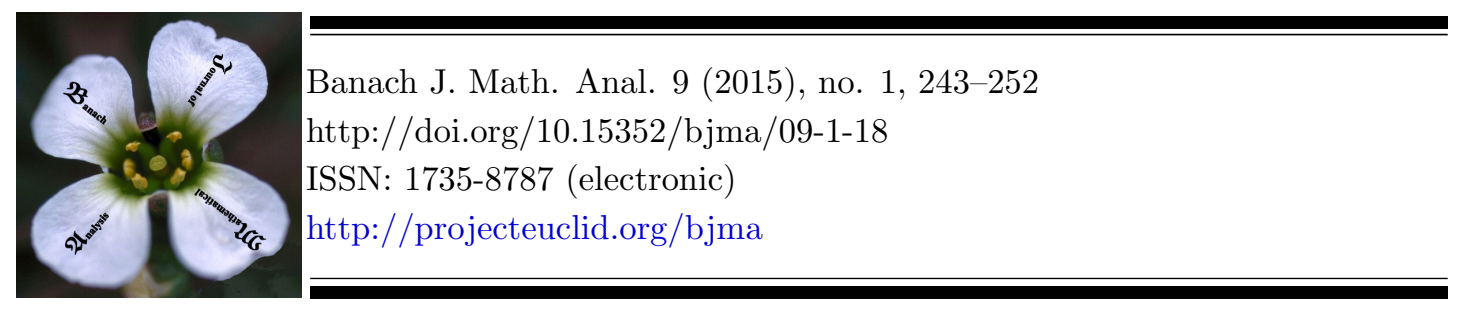

\title{
POINTS OF OPENNESS AND CLOSEDNESS OF SOME MAPPINGS
}

\author{
ĽUBICA HOLÁ ${ }^{1 *}$, ALIREZA KAMEL MIRMOSTAFAEE ${ }^{2}$ AND ZBIGNIEW \\ PIOTROWSKI ${ }^{3}$
}

Communicated by W. B. Moors

\begin{abstract}
Let $X$ and $Y$ be topological spaces and $f: X \rightarrow Y$ be a continuous function. We are interested in finding points of $Y$ at which $f$ is open or closed. We will show that under certain conditions, the set of points of openness or closedness of $f$ in $Y$, i. e. points of $Y$ at which $f$ is open (resp. closed) is a $G_{\delta}$ subset of $Y$. We will extend some results of S. Levi, R. Engelking and I. A. Vaŭnštel̆n.
\end{abstract}

\section{INTRODUCTION}

Let $X$ and $Y$ be topological spaces. Following I. $A$. Vaŭnštel̆n [21], a continuous mapping $f: X \rightarrow Y$ is called closed at $y \in Y$ if for every open subset $W \subseteq X$ containing $f^{-1}(y)$, there is a neighborhood $V$ of $y$ such that $f^{-1}(V) \subseteq W$. We denote by $C L(f)$ the set of all points of $Y$ at which $f$ is closed. Then $f$ is closed if and only if $C L(f)=Y$.

Let us recall that $f: X \rightarrow Y$ is open at $x \in X$ if it maps neighborhoods of $x$ into neighborhoods of $f(x)$ and $f$ is open at $y \in Y$ if for each open $A$ in $X$, $y \in f(A)$ implies $y \in \operatorname{Int} f(A)$. It follows from the definition that $f: X \rightarrow Y$ is open at $y \in f(X)$ if and only if it is open at each point of $f^{-1}(y)$.

Date: Received: Feb. 26, 2014; Revised: Mar. 20, 2014; Accepted: May 2, 2014.

* Corresponding author.

2010 Mathematics Subject Classification. Primary 46T20, Secondary 47H04.

Key words and phrases. Open functions, closed functions, spaces with a base of countable order, topological games. 
The investigation of the set of points of $Y$ at which $f$ is open for a continuous closed mapping $f: X \rightarrow Y$ has been studied by S. Levi in [16]. In fact, S. Levi [16] proved the following theorem.

Theorem 1.1. If $f: X \rightarrow Y$ is a continuous closed mapping on a metrizable space $X$, the set of points of $Y$ at which $f$ is open is a $G_{\delta}$ set in $Y$.

The study of the points of closedness of a continuous function and its variants has been already considered (see e.g. [18, 20]). In particular, R. Engelking [10] proved the following.

Theorem 1.2. If $f$ is a continuous mapping from a completely metrizable space $X$ into a first countable Hausdorff space $Y$, the set of points of $Y$ at which $f$ is closed is a $G_{\delta}$ set in $Y$.

The following result is due to I.A. Vaŭnštĕn [21].

Theorem 1.3. Let $f: X \rightarrow Y$ be a continuous mapping of a completely metrizable space $X$ to a first countable Hausdorff space $Y$. Then for every set $A \subseteq X$ such that $f \mid A: A \rightarrow f(A)$ is closed, there exists a $G_{\delta}$ set $B \subset X$ such that $A \subseteq B$ and the restriction $f \mid B: B \rightarrow f(B)$ is closed.

In this paper, we will generalize Theorem 1.1 for topological spaces with a base of countable order. We shall also improve Theorems 1.2 and 1.3. More precisely, we will show that Theorems 1.2 and 1.3 are true when $X$ is completely metrizable and $Y$ is a $w$-space.

Let us recall that G. Gruenhage (1976) introduced a class of topological spaces, called $W$-spaces. It is known that every first countable space is a $W$-space but the converse is not true in general $[12,17]$.

\section{OPENNESS OF MAPPINGS FROM SPACES WITH A BASE OF COUNTABLE ORDER}

We begin this section by recalling some concepts:

Definition 2.1. Let $f: X \rightarrow Y$ be a function. The function $f$ is called open (resp. feebly open) at $x \in X$ if $f(x) \in \operatorname{Int}(f(U))$ (resp. $f(U)$ has a nonempty interior) for each neighborhood $U$ of $x$. $f$ is called open (resp. feebly open) if it is open (feebly open) at each point of $X$.

Definition 2.2. A topological space $X$ is said to have a base of countable order if there is a sequence $\left\{\mathcal{B}_{n}\right\}$ of bases for $X$ such that if $x \in B_{n} \in \mathcal{B}_{n}$ and $B_{n+1} \subset B_{n}$ for each $n \geq 1$, then $\left\{B_{n}\right\}_{n}$ is a base at $x$.

Spaces having bases of countable order have been studied in depth by H. Wicke and J. Worell [22]. It is known that every metrizable space has a base of countable order.

In the following definition we will use the notion of a tree. A tree is a partially ordered set $(T, \leq)$ in which, for every $t \in T$ the set $t^{*}=\{s \in T: s<t\}$ of predecessors is well-ordered. The $\alpha$ th level of $T$ is the set of $t$ for which $t^{*}$ has order type $\alpha$. A branch in a tree is a maximal chain. A tree of the height $\aleph_{0}$ is a tree all of whose levels have cardinality less than $\aleph_{0}$. 
Definition 2.3. A sieve for a topological space $(X, \tau)$ is a pair $(G, T)$, where $(T, \leq)$ is an indexing tree of the height $\aleph_{0}$ and $G: T \rightarrow \tau$ is a decreasing function (i.e. $t \leq t^{\prime}$ implies $\left.G(t) \supseteq G\left(t^{\prime}\right)\right)$ such that

(i) $G\left(T_{0}\right)=\left\{G(t): t \in T_{0}\right\}$ covers $X$, where $T_{0}$ is the least level of $T$,

(ii) for each $t \in T, G(t)=\bigcup\left\{G\left(t^{\prime}\right): t^{\prime}\right.$ is an immediate successor of $\left.t\right\}$.

We need the following result.

Theorem 2.4. The following are equivalent:

(i) $X$ has a base of countable order.

(ii) $X$ has a sieve $(G, T)$ such that if $b$ is a branch of $T$ and $x \in \bigcap_{t \in b} G(t)$, then $\{G(t): t \in b\}$ is a base at $x$.

Proof. See [13, Theorem 6. 3].

Let $X$ be a topological space with a base of countable order and $Y$ be a topological space. Let $f: X \rightarrow Y$ be a function and $(G, T)$ be a sieve for $X$ from Theorem 2.4. Let $(T, \leq)$ be an indexing tree and for $n \in \omega$, let $T_{n}$ be the $n$th level of $T$. Define

$$
A_{1}=\left\{x \in X: \exists t_{1, x} \in T_{1}, x \in G\left(t_{1, x}\right), f(x) \in \operatorname{Int} f\left(G\left(t_{1, x}\right)\right\} .\right.
$$

Suppose that the sets $A_{1}, \ldots, A_{n-1}$ have been defined. Define

$$
A_{n}=\left\{x \in A_{n-1}: \exists t_{n, x} \geq t_{n-1, x}, t_{n, x} \in T_{n}, x \in G\left(t_{n, x}\right), f(x) \in \text { Int } f\left(G\left(t_{n, x}\right)\right)\right\} .
$$

Now, we define a function $\mathcal{O}_{f}: X \rightarrow[0, \infty]$ by

$$
\mathcal{O}_{f}(x)= \begin{cases}\inf \left\{\frac{1}{n}: x \in A_{n}\right\} & x \in A_{n} \text { for some } n \geq 1, \\ +\infty & \text { otherwise. }\end{cases}
$$

Lemma 2.5. Let $X$ be a topological space with a base of countable order and $Y$ be a topological space. A function $f: X \rightarrow Y$ is open at $x \in X$ if and only if $\mathcal{O}_{f}(x)=0$.

Proof. If $f: X \rightarrow Y$ is open at $x \in X$, then $x \in A_{n}$ for every $n \geq 1$. Thus $\mathcal{O}_{f}(x)=0$.

Suppose now $\mathcal{O}_{f}(x)=0$. We want to prove that $f$ is open at $x$. Note that for each $n \geq 1, x \in A_{n}$. Also, $x \in \bigcap_{n \geq 1} G\left(t_{n, x}\right)$. Since $\left\{t_{n, x}: n \geq 1\right\}$ is a branch of $T$, the family $\left\{G\left(t_{n, x}\right): n \geq 1\right\}$ is a base of neighborhoods of $x$. Let $U$ be an open set in $X$ such that $x \in U$. There is $n \in \omega$ such that $G\left(t_{n, x}\right) \subseteq U$. Since $x \in A_{n}, f\left(G\left(t_{n, x}\right)\right)$ is a neighborhood of $f(x)$. Thus $f(U)$ is also a neighborhood of $f(x)$.

Lemma 2.6. Let $f: X \rightarrow Y$ be a continuous function, where $X$ is a topological space with a base of countable order and $Y$ is a topological space. Then $\mathcal{O}_{f}: X \rightarrow$ $[0, \infty]$ is upper semicontinuous.

Proof. For each $n \in \omega$, define $f_{n}: X \rightarrow[0, \infty]$ by $f_{n}(x)=1 / n$, if $x \in A_{n}$ and $f_{n}(x)=\infty$, if $x \notin A_{n}$. Since $A_{n}$ is open, $f_{n}$ is upper semicontinuous. Therefore, as $\mathcal{O}_{f}(x)=i n f_{n \in \omega} f_{n}(x)$ for every $x \in X, \mathcal{O}_{f}$ is upper semicontinuous. 
Corollary 2.7. Let $X$ be a topological space with a base of countable order, $Y$ be a topological space and $f: X \rightarrow Y$ be a continuous function. The set of all points of $X$ at which $f$ is open is a $G_{\delta}$ set in $X$.

Proof. In view of Lemma 2.5, the set of all $x \in X$ at which $f$ is open equals to the set $\left\{x \in X: \mathcal{O}_{f}(x)=0\right\}$. Since

$$
\left\{x \in X: \mathcal{O}_{f}(x)=0\right\}=\bigcap_{n=1}^{\infty}\left\{x \in X: \mathcal{O}_{f}(x)<\frac{1}{n}\right\},
$$

so that the result follows from Lemma 2.6.

Recall that a function $f$ from a topological space $X$ into a topological space $Y$ is quasicontinuous at $x \in X[15]$ if for every open set $V$ in $Y$ with $f(x) \in V$ and for every open set $U$ in $X$ with $x \in U$ there is a nonempty open set $U^{\prime} \subset U$ such that $f\left(U^{\prime}\right) \subset V$. $f$ is quasicontinuous if it is quasicontinuous at every $x \in X$.

Theorem 2.8. Let $X$ be a Baire space with a base of countable order and $Y$ be a topological space. Let $f: X \rightarrow Y$ be a feebly open quasicontinuous function. Then the set of points of $X$ at which $f$ is open is a residual set in $X$ (i.e. it contains a dense $G_{\delta}$ set in $\left.X\right)$.

Proof. Let $n \in \omega$. Put $H_{n}=\left\{x \in X: \mathcal{O}_{f}(x)<1 / n\right\}$. To prove the density of $H_{n}$, let $U$ be an open nonempty subset of $X$. There must exist $k \in \omega, k>n$ and $t_{k} \in T_{k}$ with $G\left(t_{k}\right) \subset U$. Since $f$ is feebly open, $\operatorname{Int} f\left(G\left(t_{k}\right)\right) \neq \emptyset$. Put $W=\operatorname{Int} f\left(G\left(t_{k}\right)\right)$. The quasicontinuity of $f$ implies that there is a nonempty open set $L \subset f^{-1}(W) \cap G\left(t_{k}\right)$ such that $L \subset A_{k} \subset\left\{x \in X: \mathcal{O}_{f}(x) \leq 1 / k\right\} \subset H_{n}$. Also, it is easy to verify that $H_{n} \subset \overline{\operatorname{Int} H_{n}}$ for every $n \in \omega$. Thus for every $n \in \omega$, Int $H_{n}$ is dense too.

Theorem 2.9. Let $X$ be a Baire space with a base of countable order and $Y$ be a topological space. Let $f: X \rightarrow Y$ be a feebly open continuous function. Then the set of points of $X$ at which $f$ is open is a dense $G_{\delta}$ set in $X$.

Proof. By Theorem 2.8, we know that the set of points of $X$ at which $f$ is open is dense (it is residual). Corollary 2.7 implies that it is a $G_{\delta}$ set.

The following example shows that the condition of the feebly openness of $f$ in Theorem 2.9 is essential.

Example 2.10. Put $X=\{(x, 0): x \in \mathbb{Q}\} \cup\{(p / q, 1 / q): p, q$ are relatively prime, $p / q \in \mathbb{Q}\}$, where $\mathbb{Q}$ is the set of rational numbers. Consider $X$ with the topology inherited from the usual topology of the plane [1]. Let $f$ be the natural projection onto $\{(x, 0): x \in \mathbb{Q}\}$. Of course, $f$ is continuous, $X$ is a Baire metrizable space, but $f$ is not feebly open. It is easy to verify that the set of points of $X$ at which $f$ is open, is not dense in $X$.

Definition 2.11. A function $f: X \rightarrow Y$ is called irreducible if $f(X)=Y$ but for each proper closed subset $F$ of $X, f(F) \neq Y$.

Theorem 2.12. Let $f: X \rightarrow Y$ be continuous irreducible and closed. If $X$ is a Baire space with a base of countable order, then the set of points of $X$ at which $f$ is open is a dense $G_{\delta}$ set in $X$. 
Proof. By Theorem 4.10 (i) in [14], an irreducible closed function is feebly open. Thus we can use Theorem 2.9 .

Example 2.10 shows that the irreducibility of $f$ in the previous Theorem is essential.

Definition 2.13. Let $X$ be a topological space with a base of countable order and $f: X \rightarrow Y$ be a function. Define $\mathfrak{O}_{f}: X \rightarrow[0, \infty]$ by

$$
\mathfrak{O}_{f}(y)=\left\{\begin{array}{lr}
\sup \left\{\mathcal{O}_{f}(x): x \in f^{-1}(y)\right\} & y \in f(X), \\
0 & \text { otherwise. }
\end{array}\right.
$$

The following lemma follows immediately from the definition.

Lemma 2.14. Let $X$ be a topological space with a base of countable order and $Y$ be a topological space. Then a continuous function $f: X \rightarrow Y$ is open at $y \in Y$ if and only if $\mathfrak{O}_{f}(y)=0$.

Proposition 2.15. Let $X$ be a topological space with a base of countable order, $Y$ be a topological space and $f: X \rightarrow Y$ be a continuous function and closed in $y \in Y$. Then $\mathfrak{O}_{f}$ is upper semicontinuous at $y$.

Proof. If $y \notin f(X)$, then $y \notin \overline{f(X)}$ since $f$ is not closed at each point of $\overline{f(X)} \backslash$ $f(X)$. Hence $Y \backslash \overline{f(X)}$ is a neighborhood of $y$ and $\mathfrak{O}_{f}(z)=0$ for each $z \in Y \backslash \overline{f(X)}$. If $y \in f(X)$ and $\mathfrak{O}_{f}(y)=\infty$, then there is nothing to prove. Suppose that $\mathfrak{O}_{f}(y)<\varepsilon$ and choose $\varepsilon^{\prime}>0$ such that $\mathfrak{O}_{f}(y)<\varepsilon^{\prime}<\varepsilon$. Then for each $x \in f^{-1}(y)$, we have $\mathcal{O}_{f}(x)<\varepsilon^{\prime}$. Since $\mathcal{O}_{f}$ is upper semicontinuous, for each $x \in f^{-1}(y)$, we can find a neighborhood $V_{x}$ of $x$ such that $\mathcal{O}_{f}(t)<\varepsilon^{\prime}$ for each $t \in V_{x}$. Let $V=\bigcup_{x \in f^{-1}(y)} V_{x}$. Then $V$ is an open set which contains $f^{-1}(y)$. Since $f$ is closed in $y$, there is a neighborhood $W$ of $y$ such that $f^{-1}(W) \subseteq V$. If $z \in W \cap f(X)$, then

$$
\mathfrak{O}_{f}(z)=\sup \left\{\mathcal{O}_{f}(t): t \in f^{-1}(z)\right\} \leq \varepsilon^{\prime}<\varepsilon
$$

If $z \in W \cap(Y \backslash f(X))$, then $\mathfrak{O}_{f}(z)=0<\varepsilon$. Hence $\mathfrak{O}_{f}$ is upper semicontinuous.

The following theorem generalizes S. Levi's result proved for a metrizable space $X$, see [16].

Theorem 2.16. Let $X$ be a topological space with a base of countable order and $Y$ be a topological space. If a continuous function $f: X \rightarrow Y$ is closed, then the set of points of $Y$ at which $f$ is open is a $G_{\delta}$ set.

Proof. Let $E$ denote the set of points of $Y$ at which $f$ is open. Thanks to Lemma 2.14

$$
E=\left\{y \in Y: \mathfrak{O}_{f}(y)=0\right\}=\bigcap_{n \in \omega}\left\{y \in Y: \mathfrak{O}_{f}(y)<\frac{1}{n}\right\} .
$$

According to Proposition 2.15, the latter set is a $G_{\delta}$ subset of $Y$. 


\section{Closedness of COntinuous mappings}

In this section, we will study the set of points of closedness of a continuous function. This study is related to the much studied problem of the existence of Choquet kernels for set-valued mappings.

Let $\Phi: X \rightarrow 2^{Y}$ be a set-valued mapping acting between topological spaces $X$ and $Y$. We say that $\Phi$ is upper semicontinuous at $x \in X$ if for each open subset $V$ of $Y$ with $\Phi(x) \subseteq V$ there exists an open neighbourhood $U$ of $x$ such that $\Phi(U) \subseteq V$. The function $\Phi$ is called upper semicontinuous if it is upper semicontinuous at each point of $X$. Accordingly, a function $f$ maps closed sets into closed sets, if and only if the mapping $f^{-1}: Y \rightarrow X$ is upper semicontinuous everywhere.

We also define the active boundary of $\Phi$ at $x$ by

$$
\operatorname{Frac}(\Phi)(x)=\bigcap_{U \in \mathcal{U}(x)} \overline{\Phi(U) \backslash \Phi(x)},
$$

where $\mathcal{U}(x)$ denotes the set of all neighbourhoods of $x$ and we define $\Phi_{x}: X \rightarrow 2^{Y}$ by

$$
\Phi_{x}(y)= \begin{cases}\operatorname{Frac}(\Phi)(x) & \text { if } y=x \\ \Phi(y) \backslash \Phi(x) & \text { if } y \neq x\end{cases}
$$

There has been a considerable effort put into the question of when $\operatorname{Frac}(\Phi)(x)$ is a compact kernel for $\Phi$ at $x$, that is, when $\operatorname{Frac}(\Phi)(x)$ is compact and the mapping $\Phi_{x}$ is upper semicontinuous at $x$, see $[4,5,7,8]$.

In the case when the mapping $\Phi$ is strongly injective, i.e., $\Phi(x) \cap \Phi(y)=\emptyset$ for any distinct $x$ and $y$, see [3], we get the following general result.

Theorem 3.1. Let $(X, \tau)$ be a $T_{1}$ topological space and $Y$ be a Čech-complete space. If $\Phi: X \rightarrow 2^{Y}$ is a strongly injective mapping, then

$$
G=\left\{x \in X: \operatorname{Frac}(\Phi)(x) \text { is compact and } \Phi_{x} \text { is upper semicontinuous at } x\right\}
$$

is a $G_{\delta}$ subset of $X$.

Proof. Let $\beta(Y)$ be the Čech-Stone compactification of $Y$. Since $Y$ is Čechcomplete, there exists a sequence of open subsets $\left\{G_{n}\right\}_{n \in \omega}$ of $\beta(Y)$ such that $Y=\bigcap_{n \in \omega} G_{n}$. For each $n \in \omega$, let

$$
O_{n}=\bigcup\left\{U \in \tau: \overline{\Phi(U \backslash\{x\})}^{\beta(Y)} \subseteq G_{n} \text { for some } x \in U\right\}
$$

Since $\Phi$ is strongly injective, for every $x \in X$ we have $\Phi_{x}(y)=\Phi(y)$ if $y \neq x$. Clearly, each $O_{n}$ is open. Let $x \in G$ and $n \in \omega$ and $\operatorname{Frac}(\Phi)(x) \subseteq G_{n}$. There is an open set $V_{n}$ in $\beta(Y)$ such that $\operatorname{Frac}(\Phi)(x) \subseteq V_{n} \subseteq{\overline{V_{n}}}^{\beta(Y)} \subseteq G_{n}$. The upper semicontinuity of $\Phi_{x}$ at $x$ implies that there is $U \in \tau$ such that $x \in U$ and

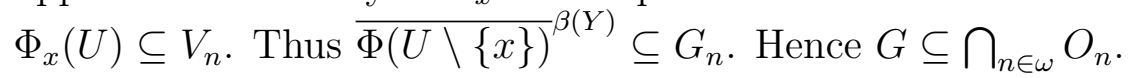


On the other hand if $y \in \bigcap_{n \in \omega} O_{n}$ and $\Phi$ is strongly injective, so that

$$
\operatorname{Frac}(\Phi)(y)=\bigcap_{U \in \mathcal{U}(y)} \overline{\Phi(U \backslash\{y\})}
$$

then $y \in G$. Thus, $G=\bigcap_{n \in \omega} O_{n}$; which is a $G_{\delta}$ set.

We can now prove the following result.

Proposition 3.2. Suppose that $f: Y \rightarrow X$ is a continuous mapping from a Cech-complete space $Y$ into a Hausdorff space $X$. If

$C L(f) \subseteq\left\{x \in X: \operatorname{Frac}\left(f^{-1}\right)(x)\right.$ is compact and $f_{x}^{-1}$ is upper semicontinuous at $\left.x\right\}$,

then $C L(f)$ is a $G_{\delta}$ subset of $X$.

Proof. Since $f$ is continuous and $X$ is Hausdorff, the graph of $f$ is closed and so $\operatorname{Frac}\left(f^{-1}\right)(x) \subseteq f^{-1}(x)$ for all $x \in X$. Therefore,

$\left\{x \in X: \operatorname{Frac}\left(f^{-1}\right)(x)\right.$ is compact and $f_{x}^{-1}$ is upper semicontinuous at $\left.x\right\} \subseteq C L(f)$ and so

$\left\{x \in X: \operatorname{Frac}\left(f^{-1}\right)(x)\right.$ is compact and $f_{x}^{-1}$ is upper semicontinuous at $\left.x\right\}=C L(f)$.

The result now follows from the previous theorem since the mapping $f^{-1}$ is strongly injective.

Corollary 3.3. Suppose that $f: Y \rightarrow X$ is a continuous mapping from a Čechcomplete space $Y$ into a Hausdorff first countable space $X$. If $Y$ has the property that every relatively countably compact subset of $Y$ has a compact closure then $C L(f)$ is a $G_{\delta}$ subset of $X$.

Proof. This follows directly from Theorem 2.3 in [4] and the previous proposition.

Many spaces satisfy the property that every relatively countably compact subset has a compact closure. For example, by the Eberlien-Šmulian Theorem, for any Banach space $(X,\|\cdot\|),(X$, weak $)$ has this property as does every Diedonné complete space, see [4] and [8].

Let us recall that G. Gruenhage [12] introduced a class of topological spaces called $W$-spaces.

Let $X$ be a topological space and $x_{0} \in X$. The topological game $\mathcal{G}\left(X, x_{0}\right)$ is played by two players $\mathcal{O}$ and $\mathcal{P}$ as follows.

In the step $n \geq 1$, the player $\mathcal{O}$ selects a neighborhood $H_{n}$ of $x_{0}$ and then $\mathcal{P}$ answers by choosing a point $x_{n} \in H_{n}$. If

$$
p_{1}=\left(H_{1}, x_{1}\right), \cdots, p_{n}=\left(H_{1}, x_{1}, \cdots, H_{n}, x_{n}\right)
$$

are the first " $n$ " moves of some play ( of the game), we call $p_{n}$ the $n^{\text {th }}$ (partial play) of the game. We say that $\mathcal{O}$ wins the game $p=\left(H_{n}, x_{n}\right)_{n \geq 1}$ if $x_{n} \rightarrow x_{0}$. We say that $\mathcal{P}$ wins the game $p=\left(H_{n}, x_{n}\right)_{n \geq 1}$ if $\left(x_{n}\right)_{n}$ does not converge to $x_{0}$.

A strategy for the player $\mathcal{P}$ is a sequence of functions $s=\left\{s_{n}\right\}$, such that $s_{n}$ is a function from $\left(H_{1}, \ldots, H_{n}\right)$ to $H_{n}$ for each $n \geq 1$. When $s=\left\{s_{n}\right\}$ is a strategy for the player $\mathcal{P}$, a $s$-play for the player $\mathcal{P}$ is a play $p=\left(H_{n}, x_{n}\right)_{n}$ such that $x_{n}=s_{n}\left(H_{1}, \ldots, H_{n}\right)$ for each $n \geq 1$. That is a play in which the player $\mathcal{P}$ 
select his (or her) choices according to the strategy s. Similarly, a strategy for the player $\mathcal{O}$ can be defined. We refer the reader to [2] for further information about other kinds of topological games and their applications in analysis.

A point $x \in X$ is called a $W$-point (respectively a $w$-point) in $X$ if $\mathcal{O}$ has (respectively $\mathcal{P}$ fails to have) a winning strategy in the game $\mathcal{G}(X, x)$. A space $X$ in which each point of $X$ is a $W$-point (respectively a $w$-point) is called a $W$-space (respectively a $w$-space.) It is known that every first countable space is a $W$-space [12, Theorem 3. 3]. However, the converse is not true in general [17, Example 2. 7].

There are $w$-spaces which are not $W$-spaces. For example [11] if $X$ is the one point compactification $T \cup\{\infty\}$ of an Aronszajn tree $T$ with the interval topology, then neither $\mathcal{P}$ nor $\mathcal{O}$ has a winning strategy in $G(X, \infty)$.

In order to prove the main result of this section, we need the following auxiliary results.

Lemma 3.4. Let $Y$ be a metrizable space and $X$ be a Hausdorff $w$-space. Let $f: Y \rightarrow X$ be continuous. If $x \in C L(f)$, then $\partial f^{-1}(x)$ is compact.

Proof. Let $d$ be a compatible metric on $Y$. If $x \in C L(f)$ is an isolated point, then $\partial f^{-1}(x)=\emptyset$. If $x \in C L(f)$ is not an isolated point, we will show that $\partial f^{-1}(x)$ is countably compact. To prove this let $\left\{y_{n}\right\}$ be a sequence in $\partial f^{-1}(x)$. Without loss of generality, we may assume that $\left\{y_{n}\right\}$ is infinite. Let $U_{1}$ be a neighborhood of $x$ and the first choice of player $\mathcal{O}$. Then we choose some $y_{1}^{\prime} \in f^{-1}\left(U_{1}\right) \backslash f^{-1}(x)$ such that $d\left(y_{1}, y_{1}^{\prime}\right)<1$. Define $x_{1}=f\left(y_{1}^{\prime}\right)$ as the answer of $\mathcal{P}$ to this movement.

In general, in the step $n$, when the partial play $\left(U_{1}, x_{1}, \ldots, U_{n}\right)$ is specified, we choose a point $y_{n}^{\prime} \in f^{-1}\left(U_{n}\right) \backslash f^{-1}(x)$ such that $d\left(y_{n}, y_{n}^{\prime}\right)<\frac{1}{n}$. Define $x_{n}=f\left(y_{n}^{\prime}\right)$ as the next move of player $\mathcal{P}$. In this way, by the induction on $n$ a strategy for the player $\mathcal{P}$ is defined. Since $X$ is a $w$-space, there is a play $p=\left(U_{n}, x_{n}\right)_{n}$ which is won by $\mathcal{O}$. Hence $x_{n} \rightarrow x$.

Let $A=\left\{y_{1}^{\prime}, y_{2}^{\prime}, \ldots\right\}$ and $W=Y \backslash A$. We claim that $W$ is not open. On the contrary, suppose that $W$ is open. Since $f^{-1}(x) \subset W$ and $f$ is closed in $x$, there is a neighborhood $U$ of $x$ such that $f^{-1}(U) \subset W$. But then $x_{n} \in U$ for infinitely many $n$. Therefore $y_{n}^{\prime} \in W$ for infinitely many $n$. This contradiction proves our claim. Let $y \in \bar{A} \backslash A$. Since $d\left(y_{n}, y_{n}^{\prime}\right)<\frac{1}{n}$ for each $n, y$ is a cluster point of $\left\{y_{n}: n \geq 1\right\} \subset \partial f^{-1}(x)$. Therefore $y \in \partial f^{-1}(x)$. This proves our result.

Lemma 3.5. Let $X$ be a Hausdorff $w$-space and $Y$ be a topological space. Let $f: Y \rightarrow X$ be a continuous mapping. Then

$$
C L(f) \subseteq\left\{x \in X: f_{x}^{-1} \text { is upper semicontinuous at } x\right\} .
$$

Proof. Let $x \in C L(f)$. We will prove that $f_{x}^{-1}$ is upper semicontinuous at $x$. Suppose that $f_{x}^{-1}$ is not upper semicontinuous at $x$. There is an open set $V$ in $Y$ such that $\operatorname{Frac}\left(f^{-1}\right)(x) \subseteq V$ and for every open neighbourhood $U$ of $x$ there is $x_{U} \in U, x_{U} \neq x$ and $y_{u} \in f_{x}^{-1}\left(x_{u}\right) \backslash V$.

Let $U_{1}$ be a neighbourhood of $x$ and the first choice of player $\mathcal{O}$. There is $x_{1} \in U_{1}, x_{1} \neq x$ and $y_{1} \in f_{x}^{-1}\left(x_{1}\right) \backslash V$. Define $x_{1}$ as the answer of $\mathcal{P}$ to this movement. 
In general, in the step $n$, when the partial play $\left(U_{1}, x_{1}, \ldots, U_{n}\right)$ is specified, there is a point $x_{n} \in U_{n}, x_{n} \neq x$ and a point $y_{n} \in f_{x}^{-1}\left(x_{n}\right) \backslash V$. Define $x_{n}$ as the next move of player $\mathcal{P}$. In this way, by the induction on $n$ a strategy for the player $\mathcal{P}$ is defined. Since $X$ is a $w$-space, there is a play $p=\left(U_{n}, x_{n}\right)_{n \in \omega}$, which is won by $\mathcal{O}$. Hence $x_{n} \rightarrow x$.

We claim that the sequence $\left\{y_{n}\right\}_{n \in \omega}$ has a cluster point. Suppose there is no cluster point of the sequence $\left\{y_{n}\right\}_{n \in \mathbb{N}}$. Thus the set $L=\left\{y_{n}: n \in \omega\right\}$ is a closed set in $Y$ and $f^{-1}(x) \subseteq Y \backslash L$. Since $x \in C L(f)$, there is an open neighbourhood $G$ of $x$ such that $f^{-1}(G) \subseteq Y \backslash L$, a contradiction.

Let $y \in Y$ be a cluster point of $\left\{y_{n}\right\}_{n \in \omega}$. Then $y \in Y \backslash V$. It is easy to verify that $y \in \operatorname{Frac}\left(f^{-1}\right)(x)$, a contradiction.

Theorem 3.6. Let $Y$ be a completely metrizable space and $X$ be a Hausdorff w-space. Let $f: Y \rightarrow X$ be a continuous mapping. Then the set of all points of $X$ at which $f$ is closed is a $G_{\delta}$ subset of $X$.

Proof. Follows from Proposition 3.2, Lemmas 3.4 and 3.5 and the fact that $\operatorname{Frac}\left(f^{-1}\right)(x)=\partial f^{-1}(x)$.

Corollary 3.7. [10, Theorem 1] For every mapping $f: Y \rightarrow X$ from a completely metrizable space $Y$ to a first countable Hausdorff space $X$, the set of all points of $X$ at which $f$ is closed is a $G_{\delta}$ set.

I.A. Vaŭnštel̆n [21] proved that if $f$ is a continuous mapping of a completely metrizable space $Y$ to a first-countable Hausdorff space $X$, then for every set $A \subset Y$ such that the restriction $f \mid A: A \rightarrow f(A)$ is closed, there exists a $G_{\delta}$ set $B \subset Y$ such that $A \subset B$ and the restriction $f \mid B: B \rightarrow f(B)$ is closed. Theorem 3.6 enables us to give the following generalization of this result.

Corollary 3.8. Let $f$ be a continuous mapping from a completely metrizable space $Y$ to a Hausdorff $w$-space $X$. Then for every set $A \subset Y$ such that the restriction $f \mid A: A \rightarrow f(A)$ is closed, there exists a $G_{\delta}$ set $B \subset Y$ such that $A \subset B$ and the restriction $f \mid B: B \rightarrow f(B)$ is closed.

Proof. Let $A \subset Y$ be such that the restriction $f \mid A: A \rightarrow f(A)$ is closed. The set $\bar{A}$ is a completely metrizable space. According to Theorem 3.6, there is a $G_{\delta}$ subset $D$ of $X$ such that $f: \bar{A} \rightarrow X$ is closed at each point of $D$. Observe that $C L(f \mid A) \subset C L(f \mid \bar{A})([9], 4.5 .13(\mathrm{a}))$. Let $D=\bigcap_{n \geq 1} G_{n}$, where each $G_{n}$ is open in $X$. By our assumption, $f(A) \subset D$. Since $f$ is continuous, $f^{-1}\left(G_{n}\right)$ is open. There is a sequence $\left\{V_{n}\right\}_{n \geq 1}$ of open sets in $Y$ such that $\bar{A}=\bigcap_{n \geq 1} V_{n}$. Thus $B=\bigcap_{n, m \geq 1} f^{-1}\left(G_{n}\right) \cap V_{m}$ has the required properties.

\section{ACKNOWLEDGEMENT}

The authors would like to thank the referee for his(her) useful comments which led to a great improvement of this paper. The second author would like to thank the Tusi Mathematical Research Group (TMRG), Mashhad, Iran. L. Holá would like to thank to grants APVV-0269-11 and Vega 2/0018/13. A. K. Mirmostafaee 
is supported by a grant from Ferdowsi University of Mashhad, No. MP92300MIM. Z. Piotrowski thanks for 2012 YSU Research Professorship Grant

\section{REFERENCES}

1. J.M. Aarts and D.J. Lutzer, Completeness properties designed for recognizing Baire spaces, Dissertationes Math. 116 (1974), 1-48.

2. J. Cao and W.B. Moors, A survey on topological games and their applications in analysis, RACSAM Rev. R. Acad. Cienc. Exactas Fs. Nat. Ser. A Mat. 100 (2006), no. 12, 39-49.

3. J. Cao and W. Moors, Quasicontinuous selections of upper continuous set-valued mappings, Real Anal. Exchange 31 (2005/2006), 63-72.

4. J. Cao, W. Moors and I. Reilly, On the Choquet-Dolecki Theoem, J. Math. Anal. Appl. 234 (1999), 1-5.

5. J. Cao, W. Moors and I. Reilly, Topological properties defined by games and their applications, Topology Appl. 123 (2002), 47-55.

6. J.J. Charatonik and K. Omiljanowski, On the set of interiority of a mapping, Glasnik Math. Vol. 17(37) (1982), 341-361.

7. S. Dolecki, Constraints, stability and moduli of upper semicontinuity, The 2nd IFAC Symposium, Warwick, 1977.

8. S. Dolecki and A. Lechicki, On the structure of upper semicontinuity, J. Math. Anal. Appl. 88 (1982), 547-554.

9. R. Engelking, General Topology, Berlin, Heldermann, 1989.

10. R. Engelking, Closed mappings on complete metric spaces, Fund. Math. 70 (1971), $103-107$.

11. J. Gerlits and Zs. Nagy, Some properties of $C(K)$, I, Topology Appl. 14 (1982), 152-161.

12. G. Gruenhage, Infinite games and generalizations of first-countable spaces, Topology Appl. 6 (1976), 339-352.

13. G. Gruenhage, Generalized metric spaces, Handbook of Set-theoretic Topology, Edited by K. Kunen and J. E. Vaughan, Chapter 10, Elsevier Science Publishers B. V., 1984.

14. R.C. Haworth and R.A. McCoy, Baire spaces, Dissertationes Math. (Rozprawy Mat.) 141 (1977), 73 pp.

15. S. Kempisty, Sur les fonctions quasicontinues, Fund. Math. 19 (1932), 189-197.

16. S. Levi, Closed mappings are open at a $G_{\delta}$ set, Portugal. Math. 38 (1979), 7-9.

17. P. Lin and W.B. Moors, Rich families, $W$-spaces and the product of Baire spaces, Math. Balkanica (N.S.) 22 (2008), no. 1-2, 175-187.

18. E. Michael, A note on closed maps and compact sets, Israel J. Math. 2 (1964), 173-176.

19. V. Mishkin, Closed mappings and the Baire category theorem, Polon. Sci. Sr. Sci. Math. Astronom. Phys. 23 (1975), no. 4, 425-429.

20. K. Morita, On closed mappings, Proc. Japan Acad. 32 (1956), no. 8, 539-543.

21. I.A. Vaŭnšteĭn, On closed mappings, Moskov. Gos. Univ. Uč. Zap. 155 (1952), no. 5, 3-53.

22. H. Wicke and J. Worrell, Characterizations of developable topological spaces, Canad. J. Math. 17 (1965), 820-830.

1 Mathematical Institute, Slovak Academy of Sciences, Štefánikova 49, 814

73 Bratislava, Slovakia.

E-mail address: hola@mat.savba.sk

2 Department of Pure Mathematics, Ferdowsi University of Mashhad, P. O. Box 1159, MASHHAD 91775, IRAN.

E-mail address: mirmostafaei@ferdowsi.um.ac.ir

3 Department of Mathematics, Youngstown state University, Youngstown, Оніо, 44555-0001, U.S.A.

E-mail address: zpiotrowski@ysu.edu 